Predicting Single-Substance Phase Diagrams: A Kernel Approach on Graph Representations of Molecules

Yan Xiang ${ }^{\dagger a}$, Yu-Hang Tang ${ }^{\dagger b}$, Hongyi Liua, Guang Lin ${ }^{\star c}$, Huai Sun ${ }^{\star a}$

aSchool of Chemistry and Chemical Engineering, Shanghai Jiao Tong

University, Shanghai 200240, China

${ }^{b}$ Lawrence Berkeley National Laboratory, Berkeley, California 94720, United

States

${ }^{c}$ Department of Mathematics \& School of Mechanical Engineering, Purdue University, West Lafayette, Indiana 47907, United States

${ }^{\dagger}$ Authors contributed equally

${ }^{*}$ Corresponding Authors

Supporting Information (SI)

S1. Hyperparameters with Finer Descriptions

The hyperparameters set described in Table 1 is denoted as $\mathrm{H} 1$. For comparison, we made another set of hyperparameters with finer descriptions up to $8^{\text {th }}$ layers of atoms, denoted as $\mathrm{H} 2$ as listed in Table $\mathrm{S} 1$.

As shown in Figure $\mathrm{S} 1$, although the maximum error in the region of small posterior uncertainty is reduced, the overall predictive ability is reduced. For one of the outlier molecules, 4-((E)-(4-propanoylphenyl)diazenyl)phenyl 
octadecenoate, the prediction is $1231 \mathrm{~K}$ using $\mathrm{H} 1$ and $1351 \mathrm{~K}$ using $\mathrm{H} 2$, the true (experimental) value is 1467 . This illustrates the difficulty to remove the tottering effect by using finer information in the graph kernel.

\section{S2. Graph Neural Network}

The graph neural network developed by Tsubaki et al. was used as a comparison in this work. The detail of this model can be found in their paper. ${ }^{1}$ and the codes to perform the graph neural network are available at https://github.com/masashitsubaki/molecularGNN smiles.

The dataset is divided into 7:1:2 for training, validation, and testing purposes. The default parameters given by Tsubaki et al. were used: radius (1), dimension (50), hidden layer (6), output layer (6), batch size (32), learning rate (1e-4), learning rate decay (0.99), decay interval (10). The whole procedure was repeated 10 times in parallel.

The MAE of the test sets is plotted as a function of the training epoch in Figure S2. The training process converged at about 400 epochs and no overfitting was observed.

\section{S3. Morgan Fingerprints}

Morgan fingerprints belong to the circular fingerprints ${ }^{2}$ family which is a refinement of the Morgan algorithm. ${ }^{3}$ ECFPs $^{4}$ and Morgan fingerprints are the representative ones. As shown in Figure S3, the substructures are created by 
cutting atoms in a given radius and transformed them into identifiers via a hash function. The final molecular fingerprint is obtained by combining all the atomic features using an indexing operation. Ignoring collisions, each bit of the final vector denotes the presence of a particular substructure. The number of occurrences of the same substructures is also considered.

In this work, the cutoff radius of the substructures was set to 2 . The molecules were encoded into 128-bit vectors. Then Gaussian process regression was applied using RBF kernel. We constrain all hyperparameters to be the same and adjust them to obtain the best prediction performance. The final length scale hyperparameter of RBF is set to 2 .

\section{S4. Predicted Properties}

Herein, we briefly introduce the properties we predicted in this work.

The critical point is identified by critical temperature $T_{c}$ (and associated critical pressure $P_{c}$ ) as a point at which gas, liquid, and superfluid coexist (red in Figure S4). According to the phase law, it is independent of thermophysical variable, therefore, nMGK is used directly.

VLE contains important information such as intermolecular interactions and free energy (enthalpy and entropy) changes of the molecule in gas and liquid phases (orange in Figure S4). According to the phase law, there is only one thermophysical variable is independent for a single-component system. The

VLE is usually described as a pair of coexistence curves of liquid and vapor 
densities as a function of temperature.

Liquid density as a function of temperature and pressure is taken as the case of two variable systems in this work (grey in Figure S4). Various thermophysical properties can be derived from the $\rho$ TP curve, such as compressibility, expansion coefficients, etc. 
Table S1. Atom and Bond Features and their Hyperparameters in MicroKernel Functions. (H2)

\begin{tabular}{ccl}
\hline features & elementary kernel & $h$ \\
\hline atomic number & atom & \\
ring size (list) & $\delta$ & 0.5 \\
ring (count) & $C$ & 0.5 \\
chirality & $\delta$ & 0.5 \\
Morgan substructure (r=3) & $\delta$ & 0.5 \\
atomic number (list, 1) & $\delta$ & 0.5 \\
atomic number (list, 2) & $C$ & 0.5 \\
atomic number (list, 3) & $C$ & 0.5 \\
atomic number (list, 4) & $C$ & 0.5 \\
atomic number (list, 5) & $C$ & 0.5 \\
atomic number (list, 6) & $C$ & 0.5 \\
atomic number (list, 7) & $C$ & 0.5 \\
atomic number (list, 8) & $C$ & 0.5 \\
hydrogen (count, 1) & $C$ & 0.5 \\
heavy atom (count, 1) & $\delta$ & 0.5 \\
heavy atom (count, 2) & $\delta$ & 0.5 \\
\hline E-Z (ring bond) & $\delta$ & 0.5 \\
\hline bond order & $\delta$ & \\
\hline & & \\
\hline
\end{tabular}



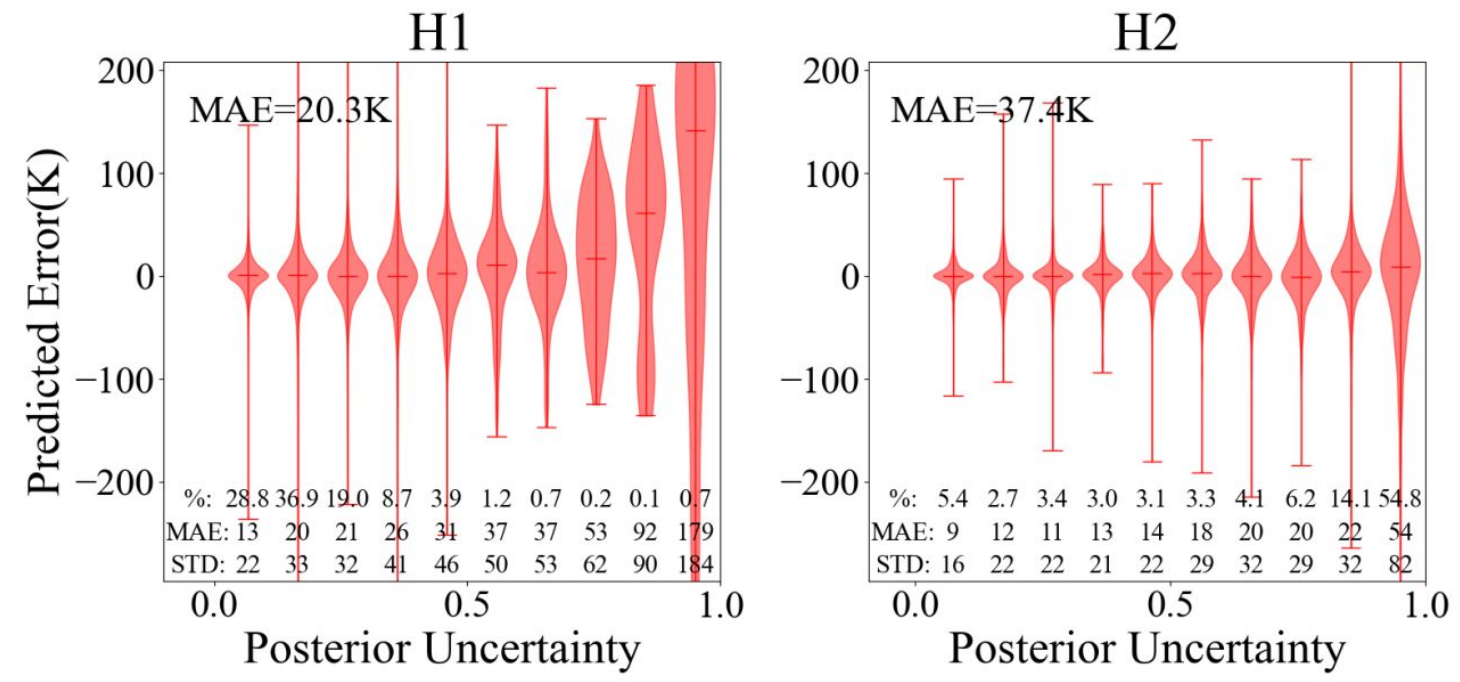

Figure S1. Relationship between predicted error and posterior uncertainty of GPR-nMGK-tMGR on critical temperature. Left: hyperparameters H1 (Table 1). Right: hyperparameters $\mathrm{H} 2$ (Table S1) 


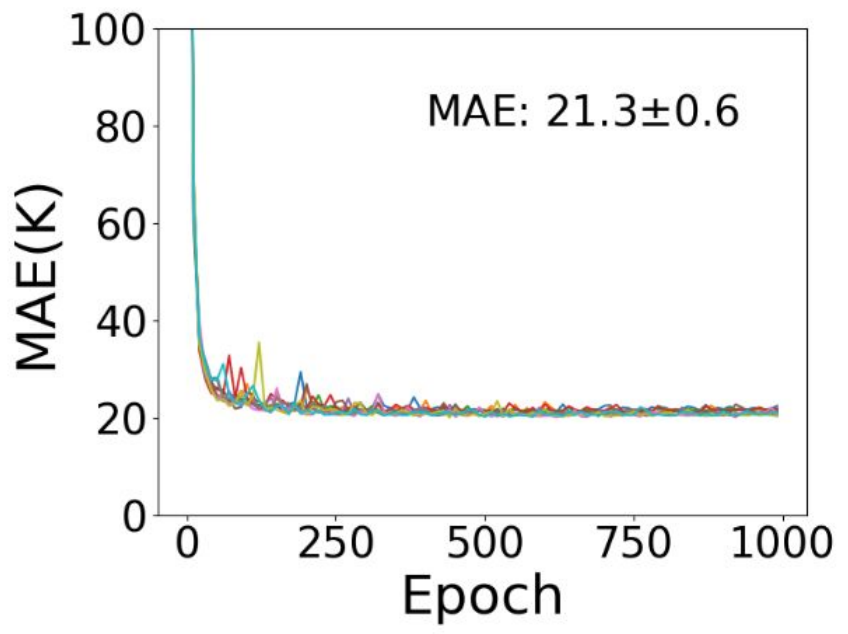

Figure S2. Change of the MAE of the test sets with the number of epochs. The learning process was repeated 10 times from different training/validation/test splitting. 
Molecule

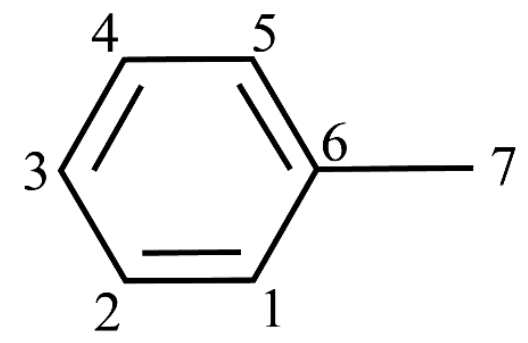

Identifier
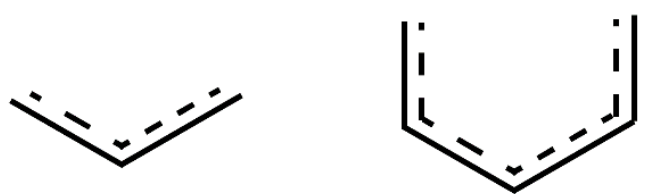

3218693969

98513984

Morgan

Fingerprint

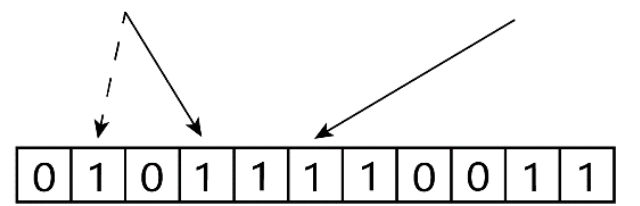

Figure S3. A representation of Morgan fingerprint and Morgan substructure fingerprint. Morgan fingerprint is a fixed-length bit vector. Because multiple substructures may be represented by the same bit code, 0 means that all corresponding features do not exist whereas 1 means that at least one corresponding feature exist. Also, substructures that exist more than once may be represented by several bit code. 


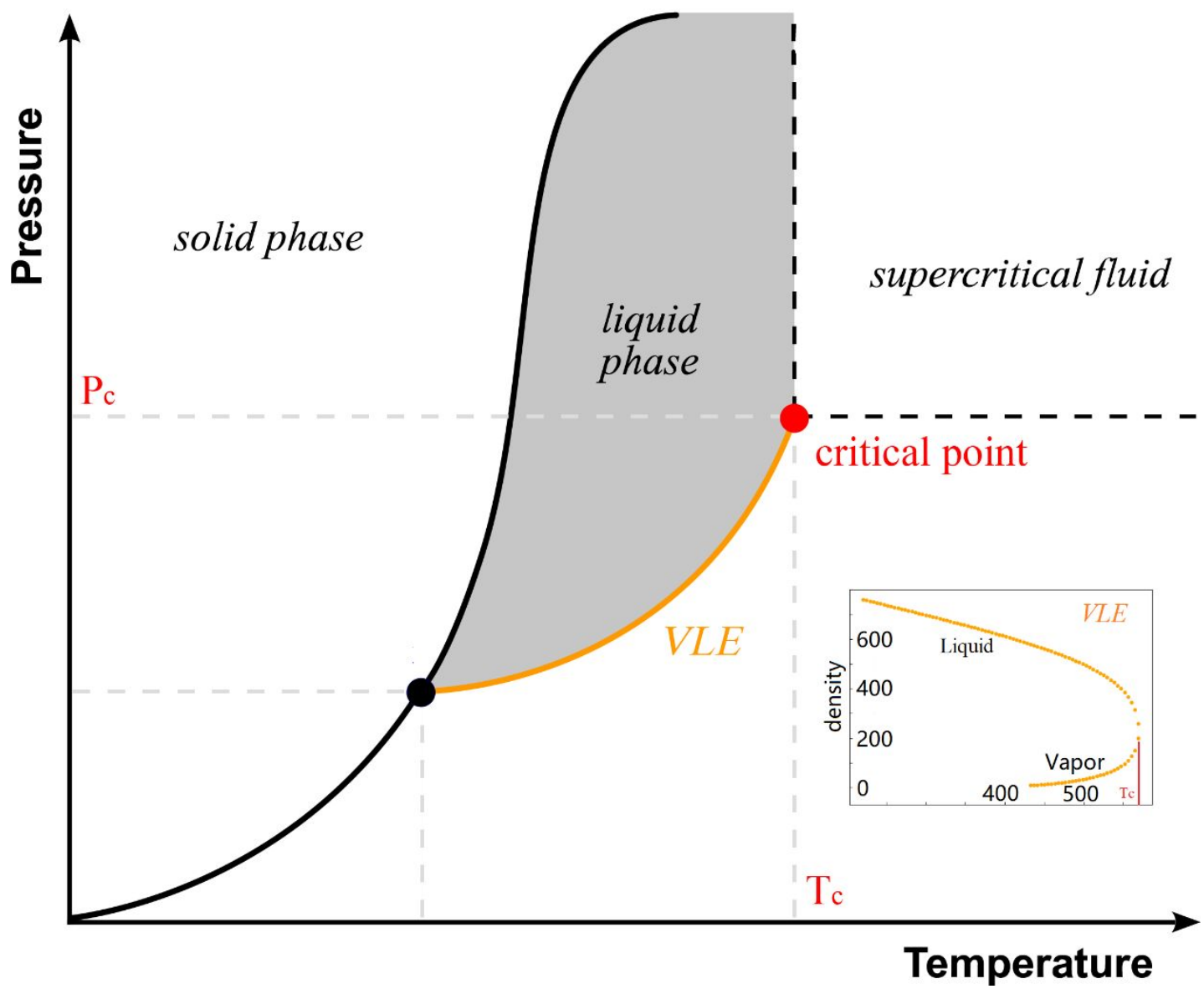

Figure S4. Typical pressure-temperature phase diagram. The properties trained in this work are colored: critical temperature (red), vapor-liquid equilibrium (orange), and liquid density (gray). 


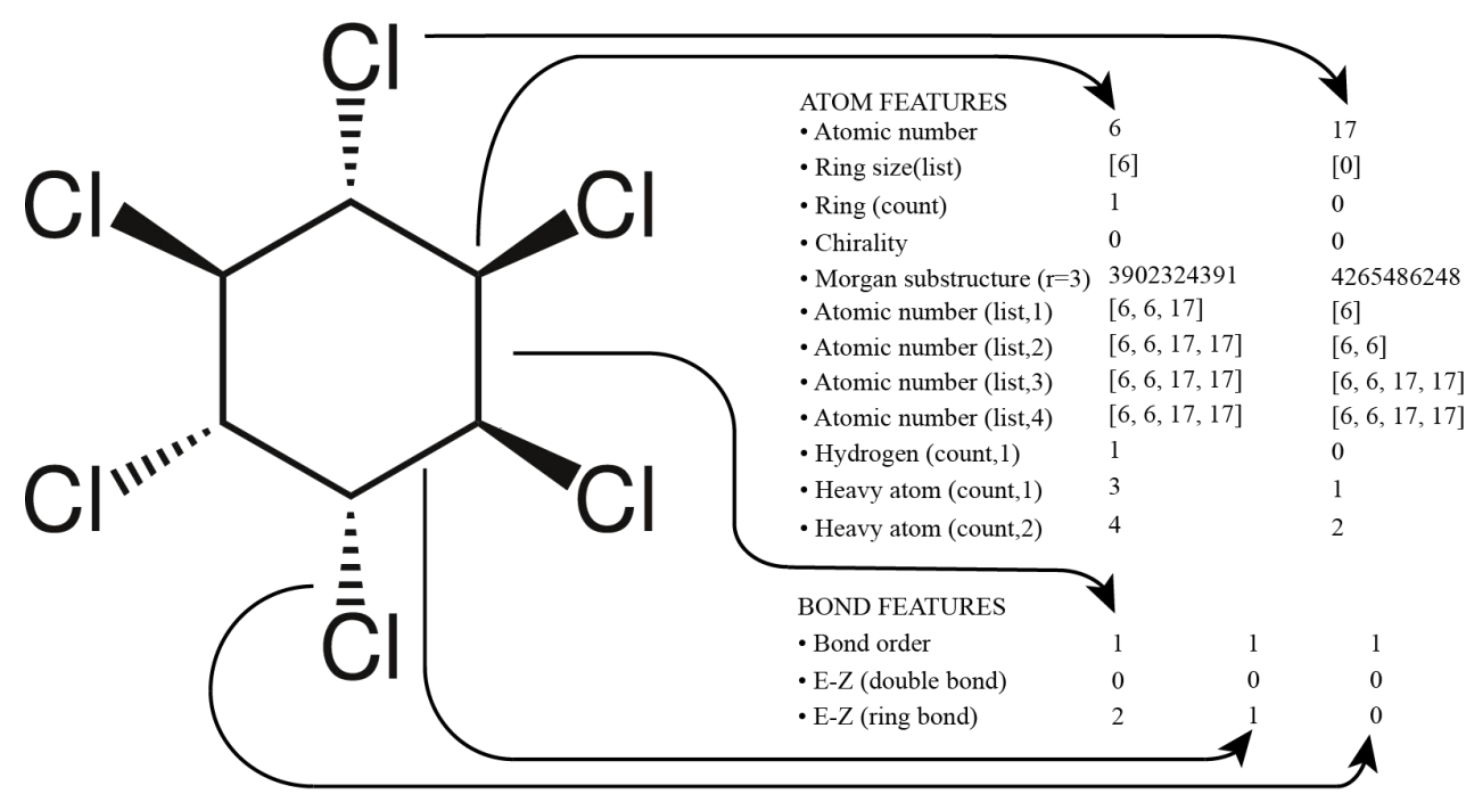

Figure S5. Atom and bond features of $\alpha$-hexachlorocyclohexane. 


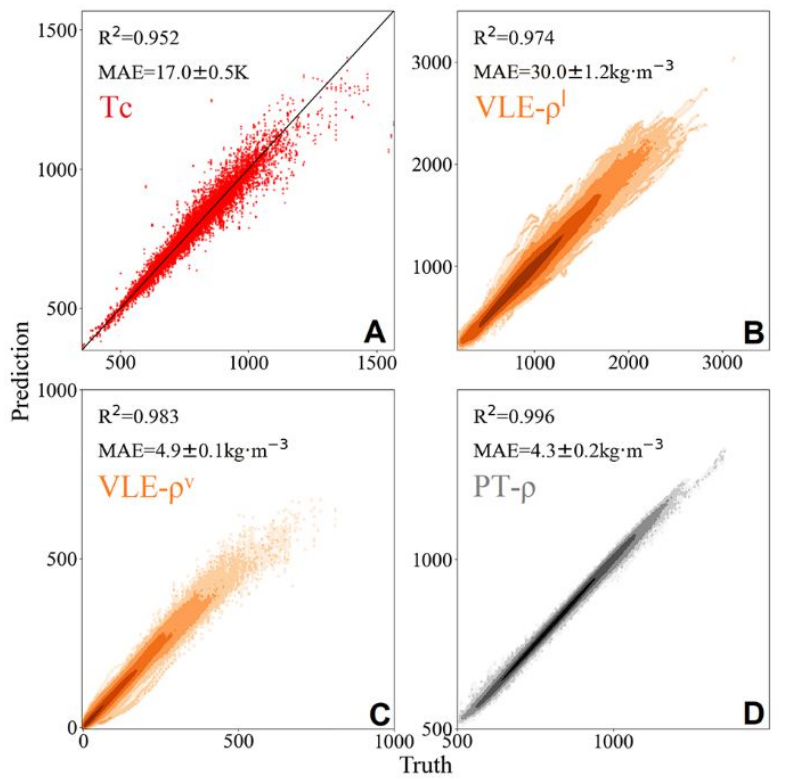

Figure S6. Relationship between the prediction and the truth value using GPRnMGK-tMGR for confident prediction with posterior uncertainty $<25 \%$. (A) Critical temperature. (B) VLE liquid density. (C) VLE gas density. (D) Liquid density. 


\section{References}

(1) Tsubaki, M.; Tomii, K.; Sese, J. Compound-protein interaction prediction with end-to-end learning of neural networks for graphs and sequences. Bioinformatics. 2019, 35 (2), 309-318.

(2) Glen, R. C.; Bender, A.; Arnby, C. H.; Carlsson, L.; Boyer, S.; Smith, J. Circular fingerprints: flexible molecular descriptors with applications from physical chemistry to ADME. IDrugs. 2006, 9 (3), 199.

(3) MORGAN, H. L. The Generation of a Unique Machine Description for Chemical Structures - A Technique Developed at Chemical Abstracts Service. J. Chem. Doc. 1965, 5, 107-112.

(4) Rogers, D.; Hahn, M. Extended-connectivity fingerprints. J. Chem. Inf. Model. 2010, 50 (5), 742-54. 